\title{
CRM TC covering paper - Robotics trends
}

\author{
Ferenc Tajti ${ }^{1,2}$, Géza Szayer ${ }^{1}$, Bence Kovács ${ }^{1}$, Balázs Dániel ${ }^{3}$, Péter Korondi ${ }^{1,4}$ \\ ${ }^{1}$ Department of Mechatronics Optics and Engineering Informatics, Budapest Univ. of Technology and Economics, Hungary \\ ${ }^{2}$ MTA-ELTE Comparative Ethology Research Group (Group number: 01 031), Hungary \\ ${ }^{3}$ Productive Programming Methods AS, Norway \\ ${ }^{4}$ MTA-BME Control Engineering Research Group, Hungary \\ korondi@mogi.bme.hu
}

\begin{abstract}
Previously, robots were mainly used by big companies in high volume manufacturing (i.e.: car industry). Industrial robots were perfect to execute procedures simplified to tiny (angular) movements. Today robots have appeared in small and medium enterprises, and they will appear rapidly in nonengineering fields as well, like offices, hospitals, and even in our homes. Robotics grains ground in newer and newer fields and it is too wide to overview it in one paper. This paper tries to summarize the nowadays robotics trends from the point of view of industrial electronics and industrial informatics.
\end{abstract}

Keywords - robotics trends; mobile robots; etho-robotics; service robots; shopfloor

\section{Introduction}

According to the report of Japanese Ministry of Economics and Trade [1] the robot industry market will be rearranged dramatically (see I). The dominance will be shifted from the classical industrial robots used in the manufacturing sector to the so called service robots, which have already overtaken the leadership in the scientific journals and conferences but their market segment was almost ignorable. It will be increased most rapidly in the near future. This perception shows the increasing trend of office, hospital, and similar robots. (In most of the cases these are mobile robots.)

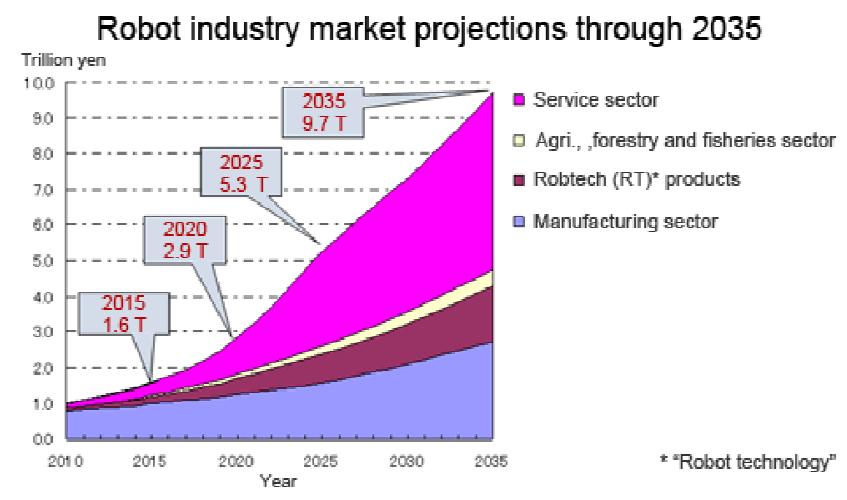

Fig. 1. Robot industry market projections [1]

Until now, the ordinary people have been able to see robots only on TV but there was no real physical contact with them. It means that robots were pure engineering stuff. They were used by engineers. It is unequivocal that industrial robots are used and programmed by robot specialist engineers. As the robots are appearing in small and middle size enterprises the robots are handled by engineers, who are not specialized in robotics. Thus the efficiency of robot programming methods must be improved in order to avoid losses caused by frequent switches in small scale production. So there is an increasing need to make the training of robots more automatized and at the same time to make them be able to fulfill more and more sophisticated tasks.

For automated material handling an industrial robot is a very handy solution, however it needs special attention in programming of work tasks. Offline programming of industrial robot is difficult because it relies on very accurate system setups and the virtual programming environment must be carefully calibrated to capture the real-life setup and to avoid any changes of the robot program on the spot itself. These problems could be avoided by using online programming of principles, but during online programming the robot is unable to produce anything. This results a continuous demand for new and effective robot teaching methods. In the field of industrial robotics the most challenging obstacle is that it takes approximately 400 times longer to program an industrial robot in complex operations than to execute the actual task [2].

In the next step the robot will be used by non engineers. In the aspect of robot users people can be divided to 4 main groups.

- robot specialist engineer

- engineer, but not robot specialist

- not engineer, but being interested in robotics

- elderly people, reluctant to robotics

For robots in our daily life it is not enough to execute a preprogrammed action line. They must be able to adopt themselves to changing environment, make their own decisions and in addition, they have to socially fit into the human environment. This requires a more sophisticated robot control method. The way of usage must be as simple (or simpler) as the usage of every office, or household devices. Service robots are designed for more sophisticated tasks than other devices, so the robot control method and the artificial intelligence must satisfy the communication tasks at a humanrobot interaction. In line with the hardware design, the low level software development this train of thought raises several additional questions like

- How should a social robot look like?

- How should we communicate with the robot?

- Is it possible for a robot to have emotions?

- What is the definition of emotions? 
People have always felt attached to their articles for personal use (phone, car, etc.). This attachment can be stronger to service robots. Turning this - currently unilateral attachment relationship - into a mutual relationship can bring not only obvious marketing advantages but enhance cooperative effectiveness in human-system interactions.

This paper is organized according to this trend starting from industrial robots and ending to social robots. In section II the human robot cooperation trends on shopfloor will be presented. The section III describes the engineering concepts for service robotics. The section IV introduces etho-robotics (ethologically inspired robotics). The $\mathrm{V}$ section is the conclusion, and the last one is the acknowledgement and references.

\section{Human Robot Cooperation on shopfloors}

While service robots are about to infiltrate everyday life still a significant effort is given to the research of robotic manipulators. As service robots become friendlier and more natural the underdevelopment of operation and programming possibilities of a manipulator becomes clear. Since small and medium enterprises (SMEs) are turning to automation the need for flexible robot cell integration is rising. Flexibility may depend on several factors:

- Hardware flexibility (robot, CNC machine)

- Integration flexibility (reconfiguration of robot cell)

- Operation flexibility (human robot interaction)

The first two components are addressed by both the robot manufacturers and informatics technology researches. The main challenge on this field is to provide a standard protocol for the different components building up the whole production system. The service oriented architecture (SOA) paradigm [3] offers a scientifically accepted approach and it is still in the main flow of interest $[4,5,6]$.

On the other hand the least flexible point of a whole system defines its overall flexibility. The problem is well defined in [7]: most flexible robot cells are sold with custom operating software and as a result the key to operational flexibility remains in the hands of the integrator. To bring more adaptively in operation [7] presents a set of robust control software. Furthermore one has to keep in mind that SMEs are often not in the position to employ highly trained operational personnel thus the easy programming and configuration contribute to flexibility (see A). Human robot cooperation is still in the focus because of the goal for simple robot programming.

\section{Robot operation in shared space}

The easiest and most natural way of programming a robot is to teach the task by hand. Moving the manipulator by hand implies danger: the robot should be energized thus a robust and safe control system is needed. Any autonomous movement of the manipulator is potentially dangerous when humans must stay or work in the reach of the robot. Controlling the human robot interaction force offers a solution and implementing impedance control [8] with use of force sensor showed feasibility of collaboration without fences. Another control scheme is presented by [9] with the comparison of PD and sliding mode controller algorithms.

The other possibility to overcome this issue is to develop compliant robot systems. The lack of robustness in previous systems prompted research for new technology. [10] Presented a control system for manipulator actuated by pneumatic muscles while [11] and [12] use direct-drive motors with wire rope mechanism.

The trend in industrial manipulator control research points clearly towards robust but compliant systems to merge accuracy and safety.

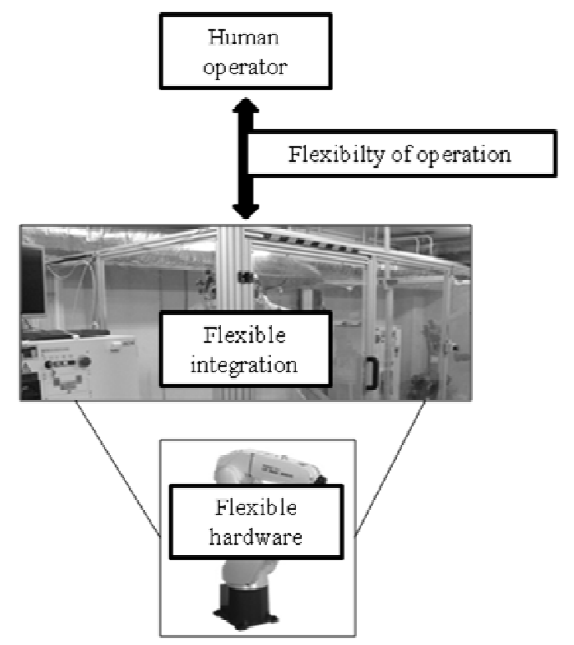

Fig. 2. Flexiblity factors

\section{Flexible human robot interaction}

In the framework of human robot cooperation the most important aspect is the bilateral communication. Physical interactions carry safety problems mentioned in the previous sub-section and environment awareness of the robot is still a significant problem. The more effective and flexible interaction with the user is intended the more sophisticated recognition technology is required.

Interesting example is presented by [13]. With traditional approach an additional channel should be used to communicate information about the object handed to the robot by the user. The use of a tactile sensor gives better potential for the robot system to understand the human intentions thus expanding the modularity of the interaction.

From the operators' point of view better understanding of the system is possible to realize through user interfaces adapted to various contributors:

- Complexity of the robot cell

- Complexity of the task performed by the robot cell

- Competence of the operator

- Information needed

The key toward better flexibility is to transform the traditional and very technical graphical user interfaces between humans 
and industrial robots (see B). The focus should be shifted from the practical operation of a robot cell towards the cognitive programming and operation. Research on this includes other fields like psychology, usability, human factors.
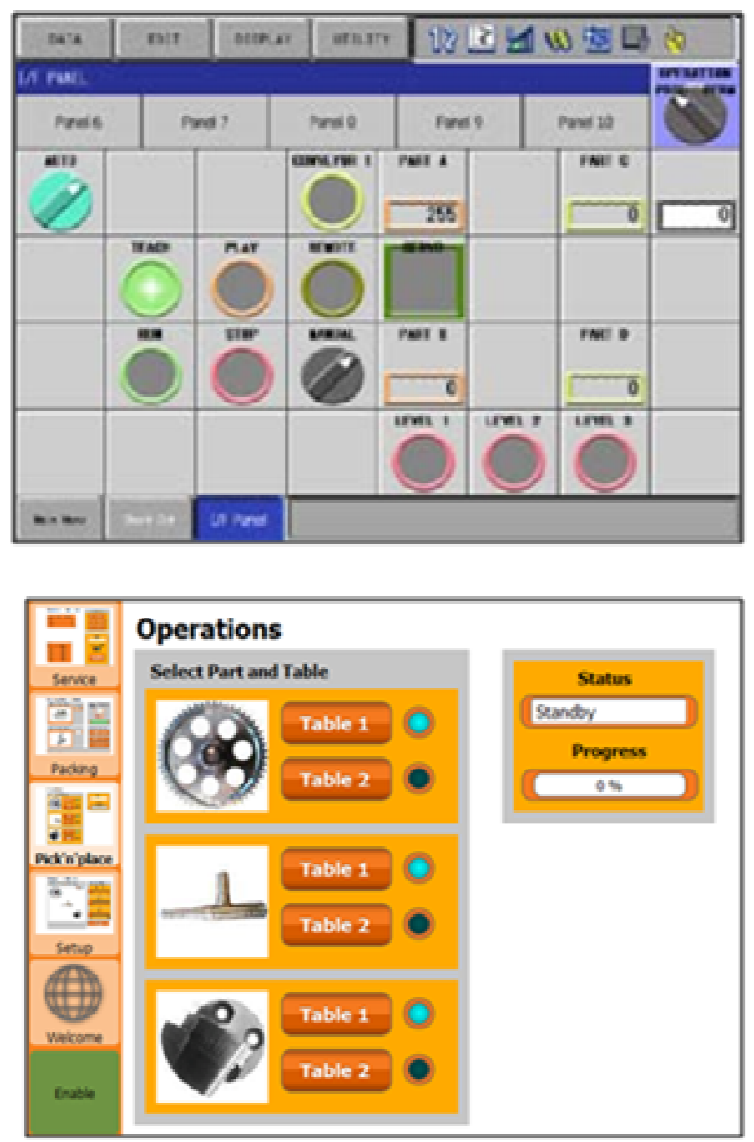

Fig. 3. Traditional (upper) and flexible (lower) user interface for industrial robots

Traditionally industrial robots are machines with as minimal human interaction as possible. Including the human in the operation not only increases the flexibility and efficiency but brings improved understanding in human robot relations and takes a new step toward better trust in automation in overall.

\section{Engineering concepts for service robotics}

Robots at the service sectors have to fulfill more and more sophisticated tasks. This is the same trend like at the evolution of industrial robots. The first industrial robot was created in 1937 by Griffith P. Taylor. [14] It contained almost only mechanical parts, and only one electric motor. It could stack wooden blocks in pre-programmed patterns. The program was on a punched paper, which activated solenoids. Nowadays industrial robots can weld, paint, mill, etc.

In a point of view the simplest robots at home are the washing machines, blenders, dishwashers, etc. These devices are created to help our daily routine tasks. The next step of this evolution is the help at our daily service type tasks. Inherently from the service tasks most of the robots in the service sector are mobile robots.

There are several responsive robot platforms can be found to help us somehow, or simply just for fun. Roomba [15], or Navibot [16] helps to clean up the household in simple and convenient way like never before. There are also some doglike equipments like AIBO [17], or Genibo but these items are just trying to act as a real dog in a very primitive level. Some human inspired robots are also available, like Honda's ASIMO [18], or SONY's QRIO [19], but these complex and technically very advanced platforms are also just scratching the surface of the real social interaction between machine and human.

For the elders there is Paro [20] or Kobie, kinds of therapy robots, which are capable to perceive light, sound, temperature and touch, thus they are capable to sense their environment and the surrounding people. These robots could interact with the user in simple ways. They could learn the preferences of the elder in behavior, and react to their names. Even "simple" toys like these could reduce the stress level of the user, help to increase the socialization between the elders and make the communications with the caregivers more flawless.

\section{Movement of the robots}

Several types of mobile robots exist like tracked, wheeled, legged, wheeled-legged, leg-wheeled, segmented, climbing or hopping. The control methods of these robots are implemented individually for every construction. In most of the cases the control algorithms use the virtual center of motion method. The path of the motion describes the path of this point [21], [22]. We can consider the whole mobile robot as a point (the centre of gravity, virtual center of motion). The velocity and the angular velocity of the centre of gravity define the motion of the robot (at constant speed ). At the path of the motion we prescribe the velocity and the angular velocity of the robot in every moment.

The most often ways for mobile robot motion are the differential type drive and the steered vehicle concept. The path planning of these mechanical constructions can be extremely difficult [23]. Indoor environments increase the number of questions in this method. (see in A.)
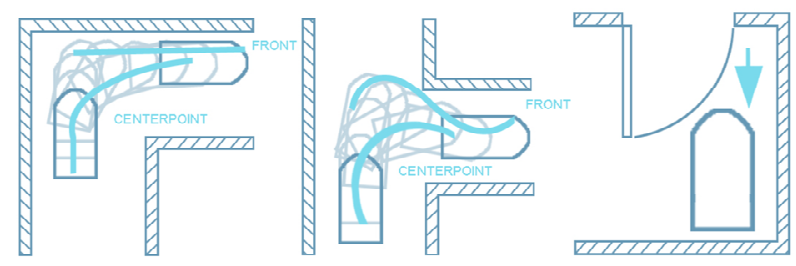

Fig. 4. Steered vehicle path planning

At different driven type mobile robots path planning is simpler, because the robot can turn around without the change 
of the position. The orientation of the robot is always the same with the moving direction. These robots (and the driven vehicles also) have only 2 DoF-s on the ground plane.

In 1994 Stephen Killough invented omni wheels. [24] These are wheels with small discs around the circumference which are perpendicular to the rolling direction. This type of wheel does not have a geometrical constrain perpendicular to the rolling direction. With this type of configuration we can get a 3 DoF holonomic system ( $x, y$, rot $z$ ). The most of the overland animals can move in 3DoF: turning and moving in one direction at the same time. The ordinary drive systems (steered wheels, differential drive) cannot perform this.

\section{Informatics concepts}

The artificial intelligence of a service robot system can be implemented on the robot, or distributed between the robot and the external environment (Intelligent Space). The Intelligent Space (iSpace) is an intelligent environment which provides both information and physical supports to humans and robots in order to enhance their performances. In order to observe the dynamic environment, many intelligent devices, which are called Distributed Intelligent Network Devices, are installed in the iSpace. [25, 26]

At the current state of science the greatest problem is caused by self-localization of robots (orientation, obstacle avoidance, path planning, etc.). There are several types of sophisticated methods for this problem. [27]

Could they be moved without requiring our presence? [28] Optical markers are generally developed for this kind of positioning. [29] (B) The most generally known optical marker is the QR code. QR code is designed only with the consideration of visual recognition and redundant information storage. (B)

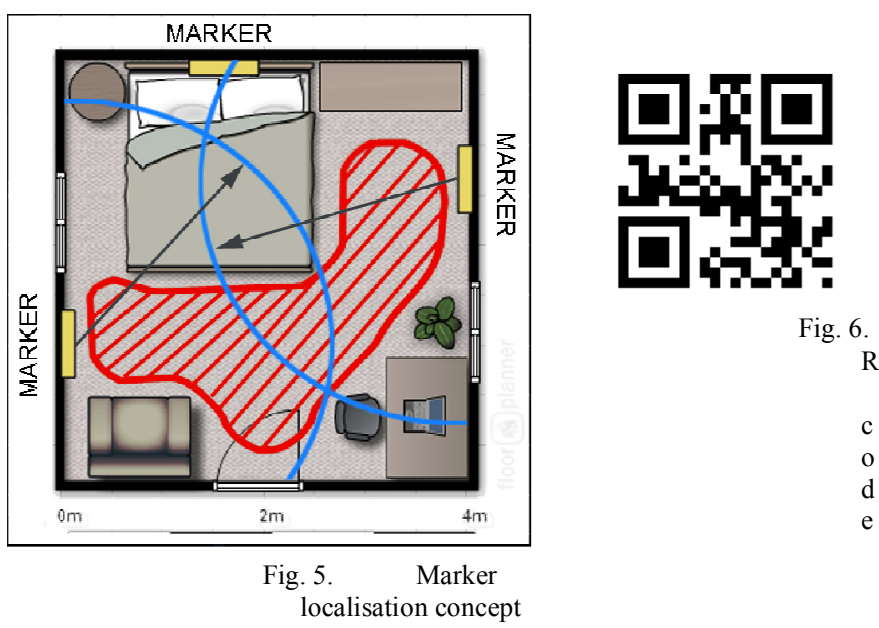

\section{etho-Robotics}

Ethology will play a major role in future development of social robotics because the emergence of new robotic agents does not only pose technological questions [30]. Only a broad synergic view of biological causality (ultimate and proximate), which has been practiced in ethology for at least half a century [31], can provide the necessary theoretical network which is able to handle future challenges.

Maybe a recent analogy could help in revealing our intentions with regard to social robotics [32], investigated the head structures of woodpeckers in order to find out how the woodpecker's head is protected against the vibration caused by drumming behaviour. The motion of a biological being has communication related senses.

According to human evolution we can usually recognize the purpose, the behavior and the mood of an animal. The goal is to enhance the artificial devices with this natural interoperability, creating a way of human-machine interactions which stands for human needs. Instead of specifying any improvised implementation based on individual intuitions, the characteristics of the etho-inspired machine behaviour has to be defined with a standardized methodology. These definitions are relying on organized observations of the natural behaviours, with a collection of perceptions. During the preorganization of these tests, the observed and analyzed behavioural variables have to be defined those can make any sense for human witness in a given scene and situation. As in many disciplines, the tests have to be performed with many subjective individuals for getting an objective result. The analyzing method consists of two simple steps: First the impressions and potential lateral information should be collected, which are caused by the main character of the test situation. Then the impressions and information have to be conjugated to the behavioural variables in a systematic way, which allows a weighted overlap between the nodes. The weight factors must be given by using the statistics of the many performed observations.

An illustrative example, when a predator animal runs for a specified target (position) in a non-straight trajectory, because of an interfering obstacle. The most of the observers can conclude the goal position while the direction of the run is not pointing to the target. Here, the essential behavioural variable is the looking direction which contains the lateral information. Using these analyzed measurements, a fuzzy model can be created that implements the initial concept. In many cases, the implementations require the use of new technologies. The solution of the animal like locomotion with different looking direction, body orientation and moving direction was induced the usage of holonomic robot platforms. A shows the above example scene, where the implemented solution can be validated by repeating the observation tests with the robot, concluding that the lateral information is reflected by the behaviour.

\section{Informatics concepts with etho-inspiration}

The best concept for the artificial intelligence is to use the abstract ethological model of the 20,000 year old human-dog relationship as the basis for the human-computing system interaction. [6] The mathematical description of the dog's attachment behavior towards its owner is an essential aspect of setting up the ethological communication model. Such approach needed an interaction among various scientific 


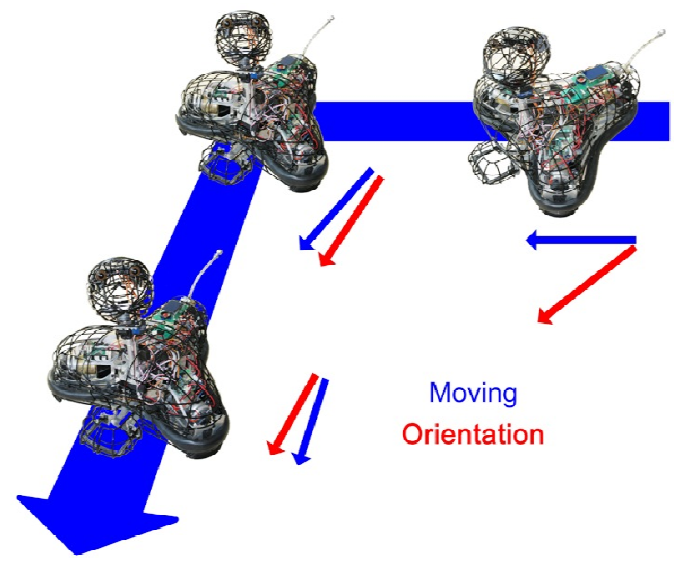

Fig. 7. Omnidirectional movement

disciplines including psychology, cognitive science, social sciences, artificial intelligence, computer science and robotics. The main goal is to find ways in which humans can interact with these systems in a "natural" way.

These systems do not have emotions, but they can act in a way that makes us believe that they are actually fond of us. While there is a need for a mathematical model of the system, the complexity of the applied solution must fit the complexity of the problem. In case of neural networks it concerns the number of the neurons and connections, in case of fuzzy logic system; there is a minimal value of the number of the fuzzy sets and fuzzy rules. Since elderly people are naturally mistrustful toward new technologies, a solution like this could make easier to use the modern devices and feel the interactions more natural. A device like this could substitute assistant dogs in some special situations, or could cooperate with the pets to serve the elderly people in need.

As it mentioned in the previous section markers are used for robot localization. Existing markers were designed to be practical and not aesthetic, thus people did not welcome them in their homes. Who would want the walls of their home or work place to be filled with QR codes? Aesthetical appearance depends on proportional harmonization of composition, tone, pattern and rhythm. Through aesthetics marker design the goal is to create harmony between human visual recognition and image processing of robots. [29] (see V) Without appropriate engineering methods and devices the information science would not be sufficient to express emotions. As it mentioned in section A, the holonomic movement is one of these ethoinspired engineering methods. The eyes of the robot are especially important at the expression of emotions. (see V) With the use of basic geometrical shapes, colors and arrangements basic emotion can be recognized.

\section{Conclusion}

Machines often can replace humans for more effective manufacturing because machines outperform humans in terms of strength, precision and endurance. Humans, however, perform better than machines when flexibility and intelligence is required.
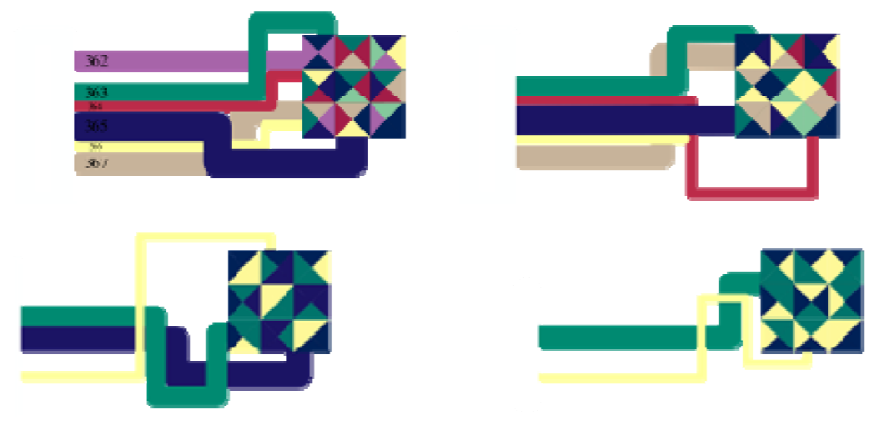

Fig. 8.

Aesthetic markers, [10]

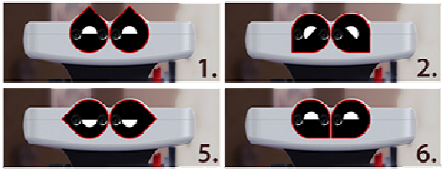

Fig. 9.

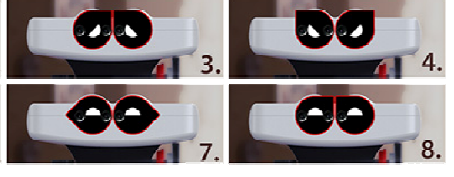

Robot eye concept

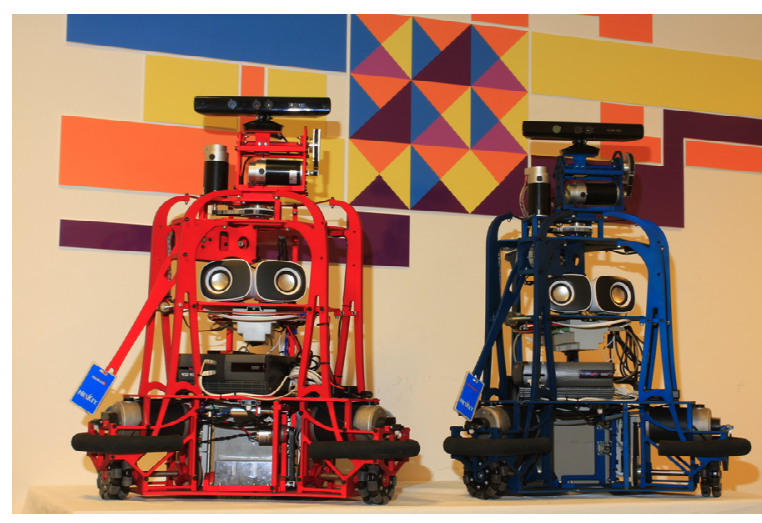

Fig. 10. Ethon robots and the aesthetic marker concept

This paper shows efficient methods and processes for teaching and optimizing complex robot tasks by introducing etho-robotics and flexible robotics. [33] Intelligent user interfaces combining information from several sensors in the manufacturing system will provide the operator with direct knowledge on the state of the manufacturing operation. Thus, the operator will be able to determine the system state quicker. Sensory system calculates and proposes the optimal process settings. The key element is the new human-machine communication channels helping the human to comprehend the information from the sensory systems.

\section{Acknowledgement}

The authors wish to thank the support of the Hungarian Research Fund (OTKA K100951), The results also discussed above are supported by the grant TÁMOP-4.2.2.B-10/1--20100009 . 


\section{References}

[1] Ministry of Economics and Trade (METI). (2010). 2035nen ni muketa robotto sangyou no shourai shijou yosoku (Market forecast of robot industry in 2035). Retrieved:

http://www.meti.go.jp/press/20100423003/20100423003-2.pdf

[2] T. Thomessen, P. K. Sannæs, and T. K. Lien, "Intuitive Robot Programming," in Proc. 35th International Symposium on Robotics, 2004.

[3] F. Jammes, H. Smit, "Service-oriented Paradigms in Industrial Automation" IEEE Trans. on Industrial Informatics, vol. 1, no. 1, pp. , Feb 2005.,

[4] G. Candido, A.W. Colombo, J. Barata, F. Jammes, "Service-Oriented Infrastructure to Support the Deployment of Evolvable Production Systems" IEEE Trans. on Industrial Informatics, vol. 7, no. 4, pp. 759 - 767, Nov 2011

[5] R. Kyusakov, J. Eliasson, J. Delsing, J. van Deventer, J. Gustafsson, "Integration of Wireless Sensor and Actuator Nodes With IT Infrastructure Using Service-Oriented Architecture" IEEE Trans. on Industrial Informatics, vol. 9, no. 1, pp. 43-51, Feb 2013

[6] M. GarciaValls, I.R. Lopez, L.F. Villar, "iLAND: An Enhanced Middleware for Real-Time Reconfiguration of Service Oriented Distributed Real-Time Systems" IEEE Trans. on Industrial Informatics, vol. 9, no. 1, pp. 228-236, Feb 2013

[7] A. Ferrolho, M. Crisostomo, "Intelligent Control and Integration Software for Flexible Manufacturing Cells ," IEEE Trans. on Industrial Informatics, vol. 3, no. 1, pp. 3-11, Feb 2007.

[8] G. Ferretti, G. Magnani, and P. Rocco, "Impedance Control for Elastic Joints Industrial Manipulators," IEEE Trans. Robot. Autom., vol. 20, no. 3 , pp. 488-498, Jun. 2004.

[9] L.M. Capisani, A. Ferrara, " Trajectory Planning and Second-Order Sliding Mode Motion/Interaction Control for Robot Manipulators in Unknown Environments" IEEE Trans. on Industrial Electronics, vol. 59, no. 8, pp. 3189-3198, Aug 2012

[10] Tae-Yong Choi, Ju-Jang Lee, "Control of Manipulator Using Pneumatic Muscles for Enhanced Safety," IEEE Trans. on Industrial Electronics, vol. 57, no. 8 , pp. 2815 - 2825 , August 2010.

[11] C. Mitsantisuk, S. Katsura, K. Ohishi, "Force Control of Human-Robot Interaction Using Twin Direct-Drive Motor System Based on Modal Space Design" IEEE Trans. on Industrial Electronics, vol. 57, no. 4, pp. 1383 - 1392, April 2010.

[12] C. Mitsantisuk, K. Ohishi, S. Katsura, "Control of Interaction Force of Twin Direct-Drive Motor System Using Variable Wire Rope Tension With Multisensor Integration" IEEE Trans. on Industrial Electronics, vol. 59, no. 1, pp. 498-510, Jan 2012.

[13] K. Suwanratchatamanee, M. Matsumoto, S. Hashimoto, "Robotic Tactile Sensor System and Applications ," IEEE Trans. on Industrial Electronics, vol. 57, no. 3, pp. 1074 - 1087 , March 2010.

[14] Griffith P. Taylor, "An Automatic Block-Setting Crane". Meccano Magazine (Liverpool UK: Meccano) 23 (3): 172. March 1938

[15] Jone J.L., "Robots at the tipping point: the road to iRobot Roomba", IEEE Robotics \& Automation Magazine, Vol. 13, Issue 1, pp. 76-78, Marc. 2006.
[16] Eric Guizzo, "Robot Vacuums That Empty Themselves", IEEE Spectrum, 16. Jan. 2012

[17] Fujita M. "On activating human communications with pet-type robot AIBO”, Proceedings of the IEEE, Vol. 92, Issue 11, Nov. 2004

[18] Sakagami Y, Watanabe R, Aoyama C, Matsunaga S, Higaki N, Fujimura $\mathrm{K}$, "The intelligent ASIMO: system overview and integration", Intelligent Robots and Systems, Vol. 3, pp. 2478-2483, 2002,

[19] Tanaka F, Suzuki H, "Dance interaction with QRIO: a case study for nonboring interaction using an entrainment ensemble model", Robot and Human Interactive communication, pp. 519-424, 2004.

[20] Shibata T, Kawaguchi Y, Wada K, "Investigation on living people with Paro at home", Robot and Human Interactive Communication, pp. 1131-1136, Toyama, 2009.

[21] Martin Udengaard, Karl Iagnemma, "Kinematic Analysis and Control of an Omnidirectional Mobile Robot in Rough Terrain" in Intelligent Robots and Systems, San Diego, pp. 795-800, 2007.

[22] H. Adachi, N. Koyachi, T. Arai, A. Shimizu, Y. Nogami, "Mechanism and Control of a Leg-Wheel Hybrid Mobile Robot" in International Conference on Intelligent Robots and Systems, Kyongju, pp. 1792-1797, 1999.

[23] S.A. Arogeti, Danwei Wang, Chang Boon Low, Ming Yu, " Fault Detection Isolation and Estimation in a Vehicle Steering System ," IEEE Trans. on Industrial Electronics, vol. 59, no. 12, pp. 4810-4820, Dec 2012.

[24] Stephen Killough, "1997 Discover Awards". Discover Magazine, Retrieved 22, September 2011.

[25] Kovács B., Szayer G., Tajti F., Korondi P. Nagy I., "Robot with Dog Type Behaviour", EDPE 2011, pp. 4, 5

[26] QinZhang,L. Lapierre, XianboXiang " Distributed Control of Coordinated Path Tracking for Networked Nonholonomic Mobile Vehicles ," IEEE Trans. on Industrial Informatics, vol. 9, no. 1, pp. 472-484, Feb 2013.

[27] D. Lee, W. Chung, "Discrete-Status-Based Localization for Indoor Service Robots," IEEE Trans. on Industrial Electronics, vol. 53, no. 5, pp. 1737-1746, Oct 2006

[28] W. Chung, S. Kim, M. Choi, J. Choi, H. Kim, C. Moon, J.-B. Song, "Safe Navigation of a Mobile Robot Considering Visibility of Environm," IEEE Trans. on Industrial Electronics, vol. 56, no. 10, pp. 3941-3950, Oct 2009.

[29] Korondi Péter, Farkas Zita, Fodor Lóránt, Illy Dániel Aesthetic Marker Design for Home Robot Localization. In: 38th Annual Conference of the IEEE Industrial Electronics Society (IECON 2012). Montreal, Kanada, 2012.10.252012.10.28. pp. 5510-5515

[30] Miklósi, Á., Gácsi, M. 2012. On the utilization of social animals as a model for social robotics. Frontiers in Psychology, 3: 75

[31] Tinbergen, N (1963). On aims and methods of ethology. Zeitschrift für Tierpsychologie, 20, 410-433.

[32] Yoon, S-H, Park S. (2011). A mechanical analysis of woodpecker drumming and its application to shock-absorbing systems. Bioinsp. Biomim. $6,1-12$.

[33] "Industrial agents: Towards collaborative production automation, management and organization," IEEE Industrial Electronics Society Newsletter, vol. 52, no. 4, pp. 17-18, 2005. 